\title{
corrosión
}

\section{bajo tensión en aceros para pretensado}

\author{
FLORENCIO DEL POZO \\ Dr. Ingeniero de Caminos \\ MANUEL ELICES \\ Dr. Ingeniero de Caminos \\ JOSE CLIMENT \\ Ledo. en C. Fisicas \\ CARLOS BARBA Dr. en C. Químicas
}

$628-3$

\begin{abstract}
sînopsing
En este trabajo se resumen los primeros resultados de un plan de ensayos, para investigar el com. portamiento frente a la corrosión de los aceros para pretensado, que está realizando el Laborato. rio Central de Ensayo de Materiales de Construcción del Centro de Estudios y Experimentación de Obras Públicas.

Los ensayos confirman el criterio de que los aceros patentados y trefilados reúnen buenas condiciones para su uso en pretensado y que el fenómeno de corrosión bajo tensión debe ser poco frecuente en la práctica, si la obra está bien realizada.

Los agentes más agresivos son los que provocan fragilización por hidrógeno; el más frecuente es el $\mathrm{SH}_{2}$. Los nitratos, citados frecuentemente como agentes típicos de corrosión bajo tensión, son menos peligrosos que el $\mathrm{SH}_{2}$. Los aceros templados y revenidos son más susceptibles a estos ataques que los aceros estirados en frío. Los cloruros han resultado menos peligrosos que los nitratos. Después de ensayos prolongados no se ha podido observar ninguna rotura por corrosión bajo tensión.

El propósito de esta investigación es lograr un ensayo sencillo y representativo de los fenómenos de corrosión bajo tensión y fragilización por hidrógeno que pueda complementar los ensayos normales que se realizan para la caracterización de un acero.
\end{abstract}

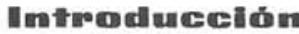

En muchas ocasiones el hombre confía su vida a la resistencia de los metales. En algunos casos, por desgracia, la estructura del metal cede bajo tensiones muy inferiores a las previstas. Estos tipos de rotura, en general, son debidos a las tres causas siguientes:

a) Fatiga: como resultado de la aplicación repetida de una pequeña tensión.

b) Fluencia: una deformación lenta a carga constante. Normalmente, se presenta a temperaturas altas.

c) Corrosión bajo tensión: una debilidad peculiar de las aleaciones en determinados ambientes químicos.

Los joyeros del siglo XIX ya se dieron cuenta del fenómeno de corrosión bajo tensión, porque sabían que las aleaciones de oro baratas se volvían frágiles al exponerlas a determinadas soluciones que contenían iones cloro.

Este fenómeno se hizo más popular, a principios de este siglo, cuando empezaron a romperse, inesperadamente, las vainas de latón de las municiones usadas por los soldados ingleses destacados en la India. Las roturas se producían, preferentemente, durante la estación lluviosa y se comprobó que eran debidas a la acción conjunta de trazas de amoníaco, contenidas en el aire húmedo, y a tensiones residuales en las vainas, producidas durante su fabricación. Si se eliminaba el amoníaco o las tensiones residuales, las roturas desaparecían. 
A este proceso, que requiere la presencia de un ambiente corrosivo y de tensiones en el material, se le llama «rotura por corrosión bajo tensión». Se sabe que la corrosión bajo tensión afecta a muchas aleaciones y ha sido la causa de la rotura de algunas estructuras pretensadas, de la explosión de calderas e, incluso, de fallos en prótesis metálicas. Este fenómeno no es privativo de los metales; se presenta en plásticos sometidos a tensión en presencia de compuestos orgánicos y también en vidrios bajo carga en presencia de vapor.

En este artículo nos limitaremos a los aceros y, en particular, a los alambres de pretensado, con los que hemos realizado la mayoría de los experimentos.

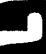

\section{Cormosión bajo tensión en aceros [teoria]}

\subsection{Corrosión bajo tensión.}

No existe una teoría capaz de explicar, de una manera unificada, el complejo fenómeno de la corrosión bajo tensión. Es difícil responder a las preguntas: ¿cómo actúa el medio ambiente para iniciar una grieta y hacer que se propague con tensiones tan bajas?, ¿por qué el mismo medio ambiente es agresivo para ciertos aceros y no lo es para otros, que apenas difieren en su composición?, ¿por qué el mismo tipo de acero es susceptible o no, según el tratamiento térmico sufrido, a la corrosión bajo tensión? La investigación en este campo ha reunido a químicos, especialistas en electroquímica, y a físicos dedicados a la metalurgía.

Ambos grupos se han planteado el problema a escala atómica, y la solución, incluso para el problema de corrosión normal, todavía está lejos, a pesar del interés económico que representa. Solamente durante el año pasado las pérdidas por corrosión ascendieron a un billón quinientos mil millones de pesetas en todo el mundo, de los cuales, quince mil millones corresponden a España.

Las teorías propuestas para explicar la corrosión bajo tensión pueden agruparse en dos tendencias:

Una de ellas se basa, únicamente, en fenómenos electroquímicos: Se hace la hipótesis de que en la aleación existen caminos muy susceptibles a la corrosión; como podrían ser los bordes de grano, por ejemplo.

Los agentes corrosivos, al alcanzar el metal, se adsorberían perfectamente en los bordes de las microgrietas y debilitarían la energía de enlace entre los átomos metálicos. Este proceso favorece la propagación de la microgrieta, porque las tensiones en los bordes son grandes, debido al efecto de entalladura y a la tensión a que está sometido el material. Esta teoría permite explicar la especificidad de los agentes corrosivos y el proceso de la corrosión en algunas aleaciones. En otras aleaciones, entre las que se encuentran los aceros, la velocidad de propagación de las grietas es demasiado grande para poderla justificar mediante una serie de reacciones químicas y demasiado lenta para atribuirla solamente a un proceso mecánico.

Esta situación ha sugerido la segunda tendencia, basada en una teoría mecánica y electroquímica. Se supone que la grieta se inicia por un proceso electroquímico igual al descrito, pero que al alcanzar un tamaño crítico se propaga una cierta distancia, debido únicamente a la concentración de tensiones (como en la rotura frágil). A continuación se inicia un nuevo proceso electroquímico seguido de una rápida rotura de origen mecánico y así sucesivamente hasta la rotura [M. Henthorne y R. N. Parkings (1966)].

En aleaciones de alta resistencia, y concretamente en aceros para pretensado, parece ser que el mecanismo más probable es el segundo. La grieta se inicia por corrosión bajo tensión y se propaga por rotura frágil. Como en las dos hipótesis se reconoce la necesidad de una corrosión electroquímica, el proceso puede detenerse mediante la protección catódica.

La protección catódica impedirá que se forme la grieta o detendrá su avance; es más, del éxito o del fracaso de la protección se podrían distinguir las roturas por corrosión bajo tensión, de otras formas de rotura. Sin embargo, en determinadas circunstancias la protección catódica puede acelerar el proceso de corrosión bajo tensión, produciéndose un fenómeno que se llama fragilización por hidrógeno.

\subsection{Fragilización por hidrógeno.}

En estos casos la grieta no se origina por un proceso de corrosión. La rotura es el resultado de la acción conjunta de la tensión y de la fragilización inducida por la penetración de hidrógeno en el metal. El mecanismo responsable de la fragilización tampoco está claro. Algunas teorías [J. Morlet, H. Johnson y A. Troiano (1958)] suponen que el hidrógeno penetra en forma atómica y se difunde hacia las imperfecciones (dislocaciones, vacantes, bordes de grano, etc.), donde se recombina en forma de hidrógeno molecular y ejerce grandes presiones. 
Estas presiones crearían cavidades submicroscópicas rodeadas de fuertes tensiones que se añadirían a las ya existentes en el material. Otro grupo de teorías [D. A. Vaughan, D. L. Phalen, C. L. Peterson y W. K. Bond (1963)] le atribuyen al hidrógeno un papel distinto. Suponen que su misión es producir tensiones dentro de la ferrita o formar hidruros metálicos que fragilizan el material.

El hidrógeno responsable de este fenómeno puede provenir de reacciones químicas que se verifiquen en el medio, o producirse debido a la protección catódica [M. H. Peterson, B. F. Brown, R. L. Newbegin y R. E. Groover (1967)]. En ambos casos, la cantidad de hidrógeno absorbida por el metal puede aumentar muchísimo cuando están presentes ciertos «venenos»; como son: los iones de azufre, de antimonio o mercurio. Se cree que estos compuestos inhiben la recombinación del hidrógeno atómico, aumentando su concentración en la superficie y, por tanto, su velocidad de difusión.

No es fácil distinguir, por la forma microscópica de la grieta, las roturas producidas por corrosión bajo tensión de las roturas por fragilización por hidrógeno. Algunos autores consideran este último fenómeno como un caso particular de la corrosión bajo tensión.

\subsection{Corrosión normal.}

Los procesos de corrosión bajo tensión y fragilización por hidrógeno raramente se dan aislados, como casos "puros»; es más, suelen ir acompañados de corrosión normal. Un caso particular de esta última, que tiene mucha importancia en aceros de pretensado, es la corrosión por picadura.

El mecanismo de corrosión es el siguiente: Por un proceso de corrosión normal, en presencia de agua y oxígeno, el acero se recubre de una capa de hidróxido básico que lo protege de ulterior corrosión (se dice que se ha pasivado). En la práctica la formación de esta capa se ve favorecida y estabilizada por la presencia de hidróxido cálcico (formado durante el fraguado y endurecimiento del cemento).

En presencia de ciertos iones $\left(\mathrm{Cl}^{-}\right.$, por ejemplo), estas capas se destruyen localmente dejando el metal al descubierto y con una diferencia de potencial respecto al resto del material protegido. Se forma lo que se llama una zona anódica, donde el metal tiene tendencia a disolverse. Como la zona es reducida, la disolución progresa en forma de pozo, formando la picadura. Si esta zona fuera extensa, a igualdad de material disuelto, los daños serían menores.

Las cavidades que se producen son grandes (véanse las figuras) y no solamente disminuyen la sección del cable, sino que, además, fragilizan el material por efecto de entalladura y lo hacen más susceptible a la rotura por fatiga, al aumentar los defectos superficiales.

\section{Corrosión bajo tensión en acemos para pretremscado [messultados experimentules]}

\subsection{Justificación de los ensayos tecnológicos.}

Es fácil darse cuenta, después de las teorías expuestas, que todavía queda un largo camino a recorrer para poder disponer de soluciones concretas a los problemas que plantea la corrosión bajo tensión.

Dado el medio agresivo, determinar la estructura y composición del acero idóneo es una pregunta que, hoy, no puede responderse con precisión. Esta es una de las tareas típicas encomendadas a una nueva rama llamada Ciencia de Materiales.

Frente a estas situaciones debe recurrirse a la experiencia real en la obra, o provocada, en el laboratorio. Existen numerosas referencias sobre el comportamiento y fallos de las estructuras pretensadas que sirven para orientar una investigación sistemática. En la primera parte del artículo de Cigna [R. Cigna, M. Maraghini y G. Schippa (1965)] se hallan bastantes referencias y breves comentarios sobre las posibles causas que provocaron la rotura de las obras.

Los ensayos realizados en el laboratorio deben reproducir las condiciones reales lo mejor posible, porque la extrapolación puede resultar muy peligrosa [H. H. Uhlig (1965)]. Por este motivo se han usado cables comerciales para pretensado, se han elegido medios agresivos que pueden aparecer en las obras $\mathrm{y}$, únicamente, se ha forzado la intensidad del ensayo.

En la interpretación de los resultados se ha tenido en cuenta el tiempo tardado hasta la rotura o hasta la aparición de la primera grieta. También se ha comprobado la pérdida de propiedades mecánicas; valor del límite elástico; carga de rotura, y ductilidad (variación de la sección transversal durante la estricción, alargamiento y número de plegados). 
Las microgrietas, provocadas por la corrosión bajo tensión, son una invitación a la rotura —como la entalladura - y, por este motivo, se han realizado ensayos de fatiga, fenómeno muy sensible a los defectos superficiales. Debido a la naturaleza integranular del ataque y a la forma alargada de los granos se producen microgrietas, casi coaxiales con el hilo, cuyo efecto se manifiesta en la prueba de torsión alternativa [como sugieren P. P. Guidugli y F. Selleri (1966)]. Por último, los ensayos se han completado con una minuciosa observación microscópica de la estructura y zonas de fractura de las probetas.

\subsection{Características del acero.}

Para la realización de los experimentos se han usado tres tipos de aceros en forma de alambre de $3 \mathrm{~mm}$ de diámetro: acero patentado y trefilado $\left(A_{1}\right)$, acero bonificado $\left(A_{2}\right)$ y acero decapado $\left(A_{3}\right)$. Veamos, a continuación, en cada caso, el proceso de fabricación, su estructura y las características mecánicas.

El acero patentado es el usado normalmente para construcciones pretensadas. En su proceso de fabricación sufre los siguientes tratamientos:

En una primera etapa (fase de patentado) el redondo procedente del alto horno se calienta por encima del punto crítico superior y, a continuación, se sumerge en un baño de plomo fundido y posteriormente se enfría en el aire. Como resultado de este tratamiento, el acero tiene una estructura sorbítica apta para la deformación plástica.

En una segunda etapa se procede a la eliminación de óxidos, superficies y cascarilla, procedentes del tratamiento térmico, mediante un decapado en baños ácidos. Luego se protege la superficie mediante una capa de fosfatos (bonderizado) y un encalado, cuya finalidad es servir de lubricante en la hilera y proporcionarle protección contra la corrosión.

Por último, en la tercera etapa, el material sufre una reducción de sección al pasar a través de la hilera. Durante este proceso el acero se agría, debido al endurecimiento por deformación, y no quede apto para nuevas reducciones, por lo que se le somete, nuevamente, a todo el ciclo descrito. Después de una serie de ciclos el alambre se endereza y envejece, antes de su expedición.

El acero bonificado tiene una historia térmica distinta. Una vez que se le ha dado, en caliente, la forma definitiva, se le somete a un temple entre las temperaturas de $800^{\circ}$ y $900^{\circ} \mathrm{C} \mathrm{y}$, posteriormente, a un revenido entre $400^{\circ}$ y $500^{\circ} \mathrm{C}$. En el acero patentado y trefilado las propiedades mecánicas se han obtenido por trabajo en frío, mientras que el acero bonificado ha adquirido sus propiedades mediante un tratamiento térmico. Por último, se han usado aceros patentados y trefilados sin la capa de protección para contrastar la eficacia de la misma frente a los medios agresivos.

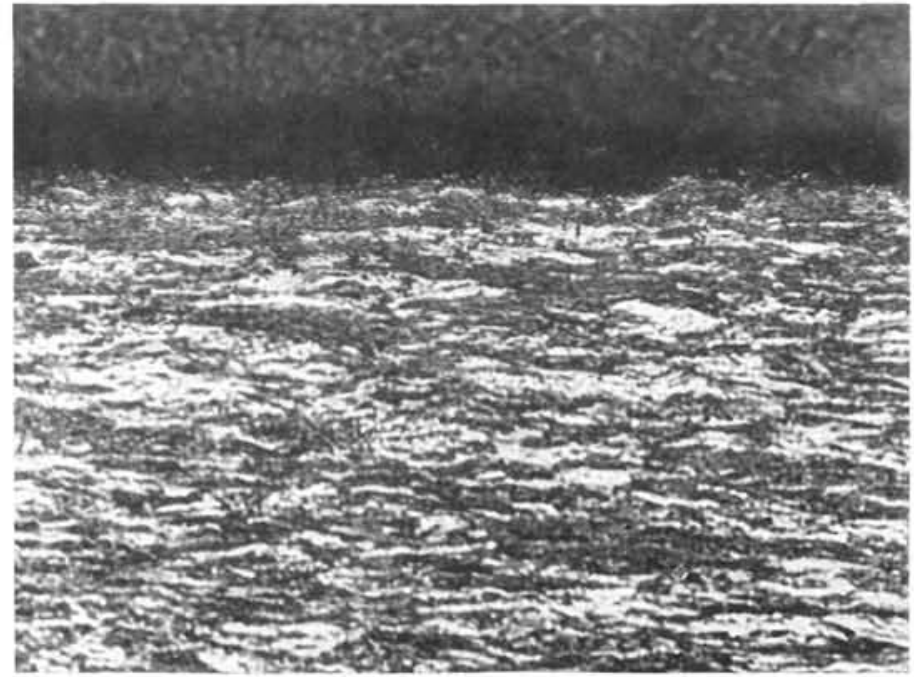

Fig. 1 a. Estructura de un alambre patentado $y$ trefilado en frío: Obsérvese la orientación preferente del grano en la dirección del eje y la superficie relativamente lisa del borde, en comparación con la figura $1 \mathrm{~b}$. Aumentos $\times 300$.

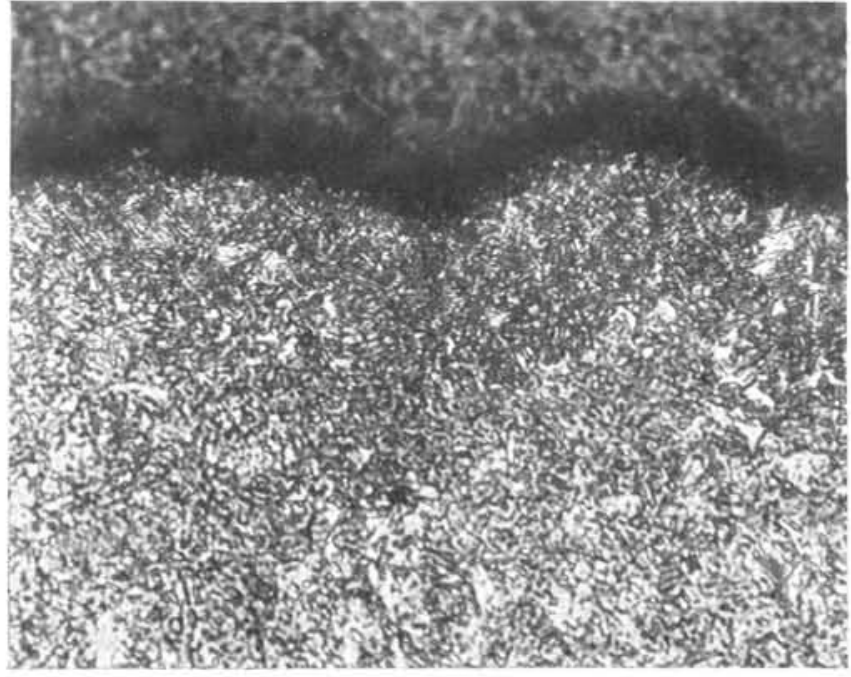

Fig. 1 b. Estructura de un alambre templado $y$ revenido (bonificado): Obsérvese que no hay orientación preferente y que la superficie del borde es más rugosa que en la figura 1 a. Aumentos $\times 300$. 


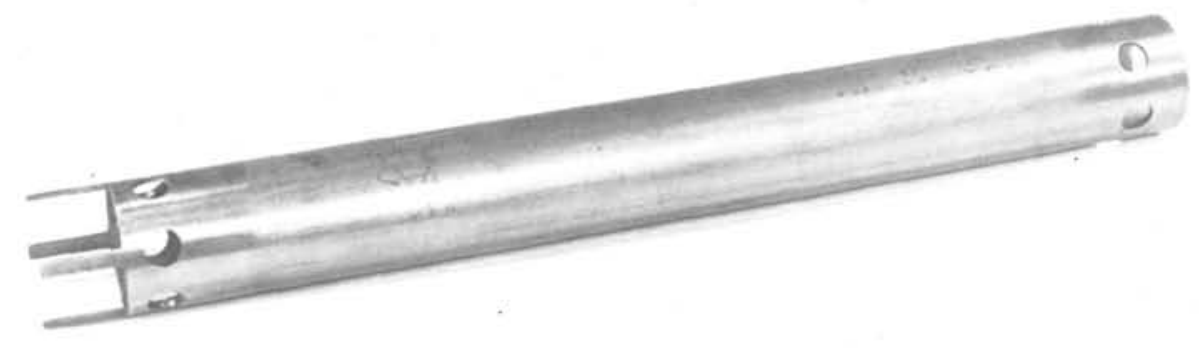

Fig. 2. Célula de corrosión: La dis posición del alam. bre de pretensado es coaxial. Los orificios laterales permiten la salida de los gases. El tubo, de acero inoxidable, se sitúa coaxialmen. te dentro de la cé. lula para establecer y controlar la dife. rencia de potencial entre el medio agre. sivo y el alambre.

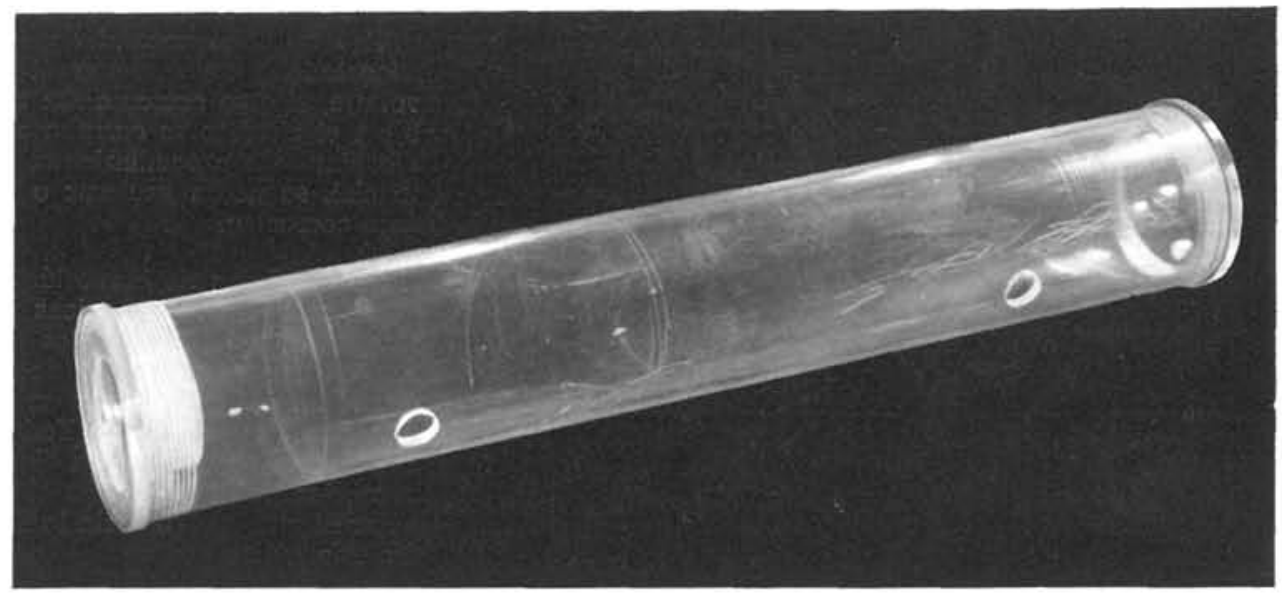

La diferencia fundamental entre los aceros patentados y trefilados y los bonificados reside en su estructura y estado de la superficie. Los primeros tienen sus granos alineados en la dirección del trefilado y una superficie relativamente lisa (fig. 1 a), mientras que la estructura de los aceros bonificados no muestra ninguna orientación preferente y su superficie es más rugosa (fig. $1 \mathrm{~b}$ ).

Esta diferencia de estructuras se manifiesta en el comportamiento mecánico, especialmente en las pruebas de plegado. Los alambres bonificados, debido a la irregularidad de su superficie, resisten menos ciclos que los patentados y trefilados; sin embargo, cuando han sufrido un revenido fuerte son menos susceptibles a la fragilización. La diferencia de estructuras también influye en el comportamiento químico.

Los aceros bonificados son más susceptibles a la corrosión bajo tensión, fenómeno que se manifiesta porque en los ensayos realizados los tiempos de rotura son más cortos y la resistencia a la fatiga es mucho menor. Se supone que una de las ventajas de los aceros patentados y trefilados frente a la corrosión bajo tensión (recordemos que es una corrosión intercristalina) es que, debido a la forma alargada de sus granos, el tanto por ciento de materia intercristalina susceptible al ataque es menor en los aceros bonificados.

\subsection{Características del medio.}

En los experimentos de laboratorio tratamos de aclarar si los alambres son atacados, y en qué medida, por agentes que pueden encontrarse en obra: agua del mar, agua con sustancias orgánicas en suspensión, agua estancada, yesos, abonos, atmósferas que contengan sulfhídrico, anhídrido carbónico, etc. Incluso una atmósfera húmeda y, en ciertas circunstancias, el agua destilada puede causar roturas por corrosión bajo tensión en aceros de alta resistencia [M. Brachet, F. Duffaut y P. Lacombe (1967)].

Para los ensayos se han seleccionado los siguientes medios: agua de mar, agua de cal y agua de yeso; soluciones con cloruro cálcico; soluciones con nitrato $-60 \%$ de $\left(\mathrm{NO}_{3}\right)_{2} \mathrm{Ca}$ y $4 \%$ de $\mathrm{NO}_{3} \mathrm{NH}_{4}-$ y soluciones con sulfhídrico.

Las células de corrosión utilizadas para los ensayos se indican en la figura 2. Las zonas del alambre que no debían ser expuestas a la corrosión iban recubiertas de una capa protectora de pintura para evitar 


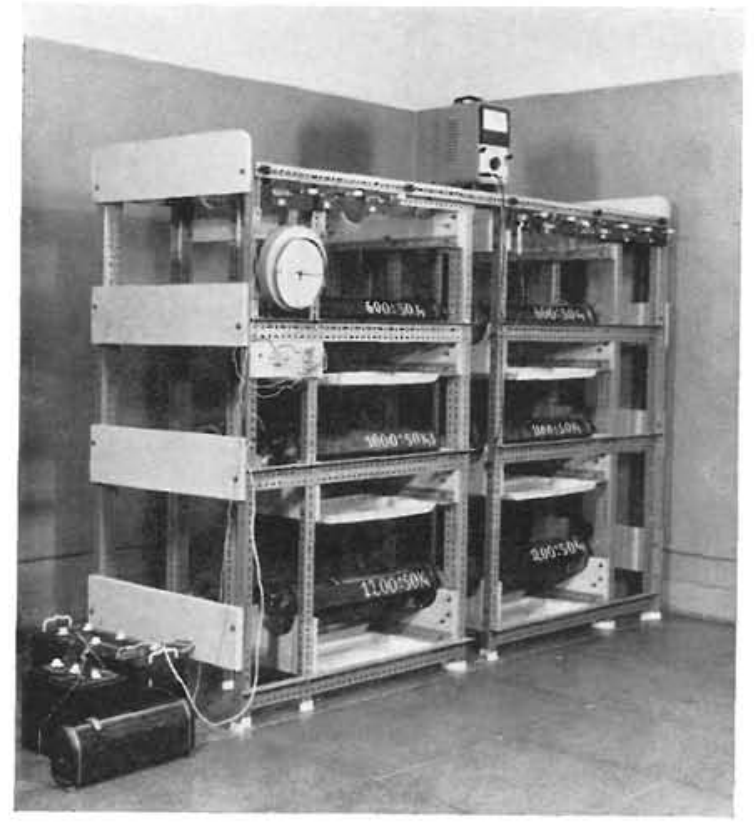

y se han hecho ensayos de inmersión periódica con un montaje más simple (fig. 4), sugerido por Bukowiecki. En algunos casos (soluciones con nitratos), el ataque periódico es menos agresivo que el de la inmersión continuada, pero en otros (soluciones con $\mathrm{ClNa}$ ) es más peligroso, debido al mayor aporte de oxígeno. Por último, queremos resaltar que no se han reseñado experiencias con barras embebidas en hormigón, o mortero de cemento, en parte porque estos ensayos no se han terminado y porque los resul. tados deben ser análogos a los que se obtienen con el medio agresivo sin proteger el cable con hormigón [Baümel y Engell (1959)].

\subsection{Resultados experimentales.}

A continuación comentamos los primeros resultados obtenidos de un plan de ensayos que realiza el Laboratorio Central para investigar el comportamiento frente a la corrosión de los aceros para pretensado.

Consideremos, en primer lugar, el efecto producido por el CINa. Los valores de la carga de rotura apenas han variado después de un ensayo cuya duración ha oscilado entre 1 y 3 meses y el material se ha sometido a distintas tensiones, hasta alcanzar el límite elástico (fig. 5). La pequeña disminución que aparece en algunos casos se cree que es debida a una mayor aireación de la muestra, lo que implica un aumento de la capa de óxido y una disminución de la sección eficaz.

La ductilidad se ha tenido en cuenta a través del ensayo de

el par diferencial. Para controlar la diferencia de potencial entre el medio y el cable se conectado a un extremo de una fuente de voltaje, el otro extremo iba conectado al cable y el circuito se cerraba a través del medio. Por este procedimiento, variando el potencial, se podía acelerar o detener el proceso corrosivo.

Algunas células de corrosión llevan incorporadas resistencias eléctricas para regular la temperatura del medio. Los ensayos se realizan en grupos idénticos de ocho unidades, con el fin de poder hacer un estudio estadístico con los resultados. Todas las unidades están unidas mediante un dispositivo electromecánico que interrumpe el ensayo al romperse el primer cable. En la figura 3 se muestra uno de los grupos.

En algunas situaciones, la inmersión conti.

政

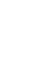

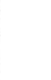


plegado standard. El número de ciclos en función de la tensión de los alambres se ha representado para distintos tiempos en la figura 6. Para los aceros patentados y trefilados la influencia ha sido muy pequeña; sin embargo, es fácil apreciar una fragilización notable en los aceros bonificados. A la vista de los resultados, y de la cantidad y distribución de las picaduras, parece ser que los distintos tipos de alambres tienen, aproximadamente, la misma tendencia a la corrosión. Sin embargo los alambres bonificados deben considerarse más peligrosos porque son más sensibles a las entalladuras (como lo demuestra el ensayo de plegado).

En los alambres patentados y trefilados, debido a la estructura alargada de sus granos, les es difícil a las grietas progresar normalmente a la dirección del eje, produciéndose una picadura peculiar, en forma de escamas,

como puede apreciarse perfectamente en la figura 7, donde, además, se ve el efecto del tiempo en la variación del tamaño de la picadura. Como contraste se muestra la forma de la picadura en un acero bonificado (fig. 8), donde la falta de orientación de los granos no puede impedir el progreso de la corrosión hacia el centro.

El comportamiento de los dos tipos de acero es distinto frente a los nitratos. Todos los aceros bonificados rompieron por corrosión bajo tensión durante el ensayo, mientras no rompió ninguno de los aceros patentados y trefilados (tabla I).

La forma de la rotura por corrosión bajo tensión, rotura frágil, puede apreciarse en la figura 9, donde se compara con la rotura ductil, producida en un ensayo a tracción de un acero patentado y trefilado sometido al mismo ataque químico.

En la figura 10 se muestra una grieta por corrosión bajo tensión debida al ataque de nitratos en un acero bonificado.

Los resultados del ensayo de plegado se indican en la figura 11, resaltando, una vez más, la tendencia a la fragilización de los aceros bonificados.

En la figura 12 se resume el comportamiento mecánico de unos cables patentados y trefilados que no han mostrado síntomas de corrosión bajo tensión después de un prolongado ataque con nitratos.

Los ensayos de fragilización por hidrógeno, debidos al sulfhídrico, son más espectaculares.

Los cables que mejor resistieron fueron los patentados y trefilados; a continuación, los mismos sin protección superficial y, por último, los bonificados. La prueba de plegado se indica en la figura 13; también se incluyen los valores obtenidos con alambres sin tensión para mostrar que el efecto fragilizador del hidrógeno no depende de la carga.

Sin embargo, el tiempo de rotura sí depende de la tensión, como se mues. tra en la figura 14.

El tipo de rotura causada por el $\mathrm{SH}_{2}$ es frágil y análoga al de corrosión bajo tensión producida por los nitratos. Compárense las figuras 9 y 15 .

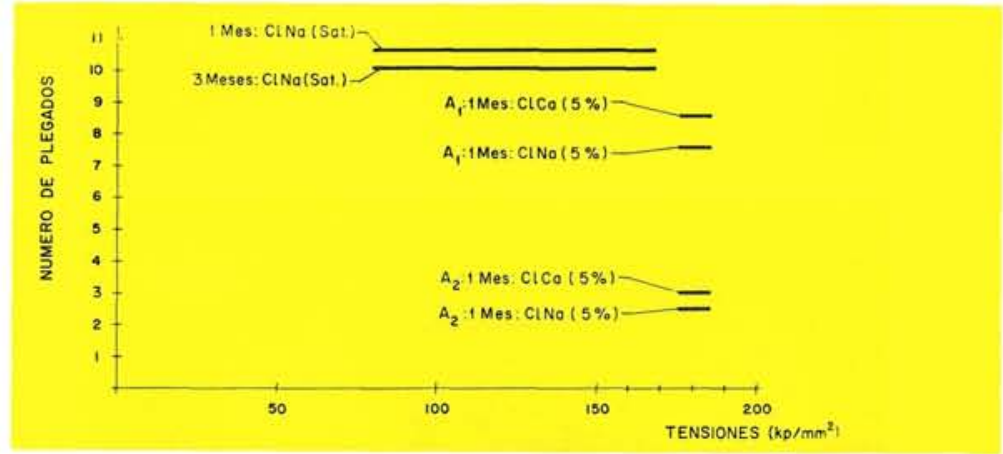

Fig. 6. Efecto de los cloruros y de la tensión en la ducti. lidad: Para aceros patentados $y$ trefilados la influencia ha sido pequeña; sin embargo, es fácil apreciar una fragilización notable en los aceros bonificados. 
Fig. 7. Corrosión por cloruro sódico en alambres patentados y tre filados: El tipo de corrosión es en forma de escamas. El tiempo de permanencia en el medio agresivo ha sido 1 mes en la figura de la izquierda y de 3 meses en la figura de la derecha, Aumentos $\times 300$.
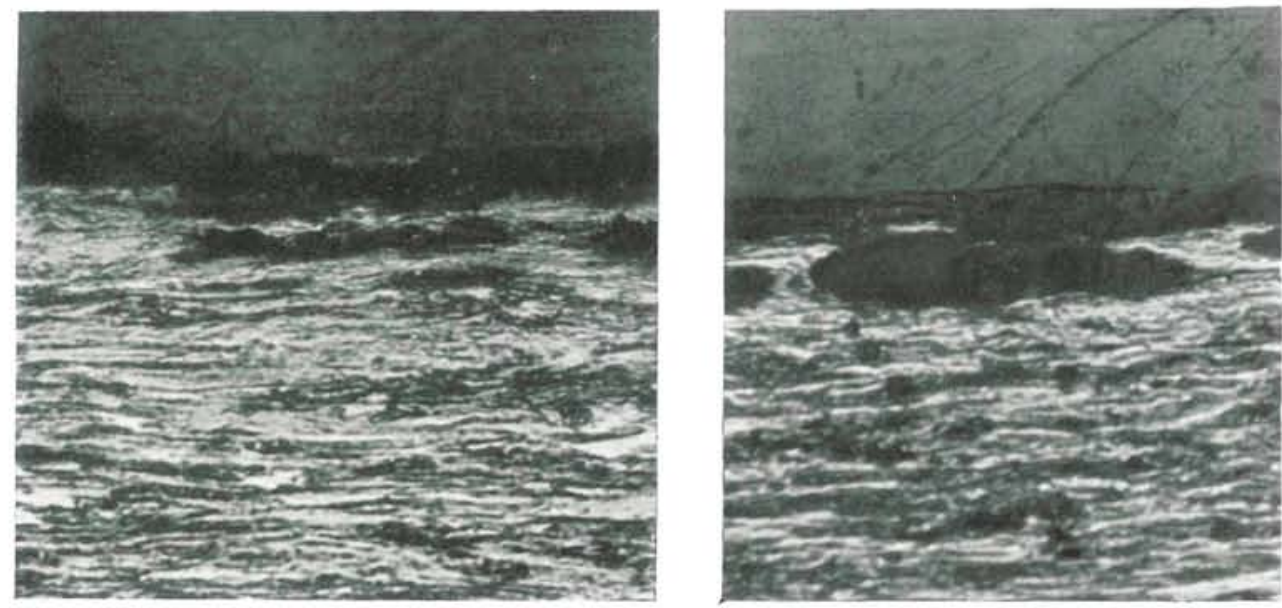

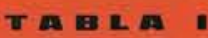

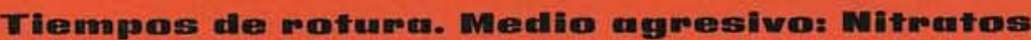

Tipo de ensayo

1. Temperatura ambiente. $>$ de 2 meses Acero: $A_{1}$.

Tensión, $170 \mathrm{kp} / \mathrm{mm}^{2}$.

2. Temperatura, $100^{\circ} \mathrm{C}$. $>$ de 2 meses Acero: $A_{1}$.

Tensión, $170 \mathrm{kp} / \mathrm{mm}^{2}$.
Tipo de ensayo

3. Temperatura, $100^{\circ} \mathrm{C}$. Acero: $A_{1}$.

Tensión, $180 \mathrm{kp} / \mathrm{mm}^{2}$.

Ciclos de inmersión de 1 hora semanales.

4. Temperatura, $100^{\circ} \mathrm{C}$. Acero: $A_{2}$.

Tensión, $180 \mathrm{kp} / \mathrm{mm}^{2}$. Ciclos de inmersión de 1 hora semanales.
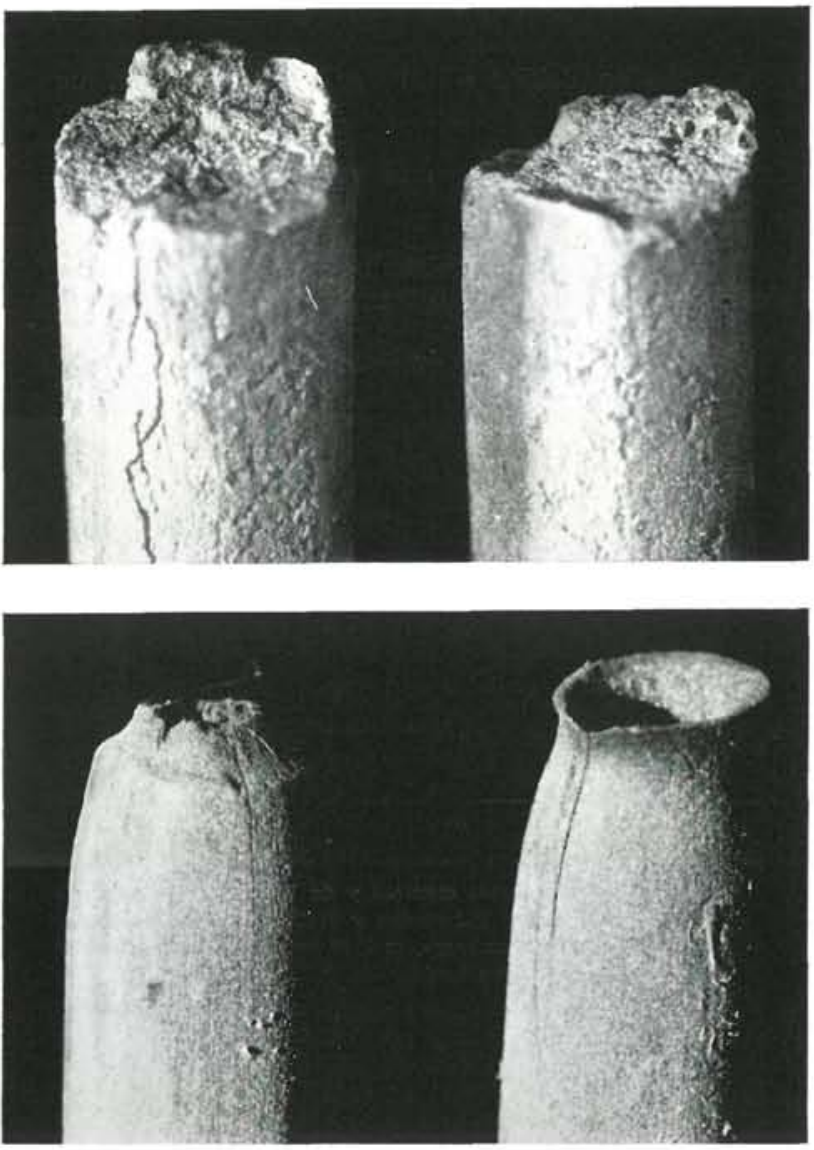

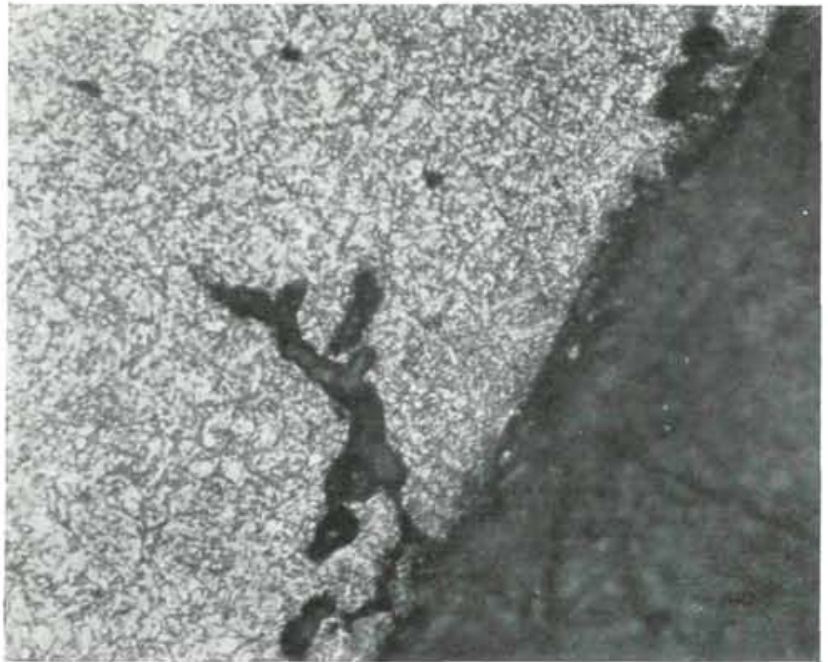

Fig. 8. Corrosión, por ClNa, en alambres templados y revenidos: El tipo de corrosión es una picadura típica, debido a la falta de orientación de los granos. Tiempo de permanencia, 1 mes. Aumen. tos $\times 300$.

Fig. 9. (Superior): Acero templado y revenido: Rotura frágil, por corrosión bajo tensión debida a nitratos. Aumentos $\times 12$ - (Infe rior): Acero patentado y trefilado: Rotura dúctil (provocada en una máquina de tracción) después de estar sometido al mismo ambiente agresivo. Aumentos $\times 12$. 


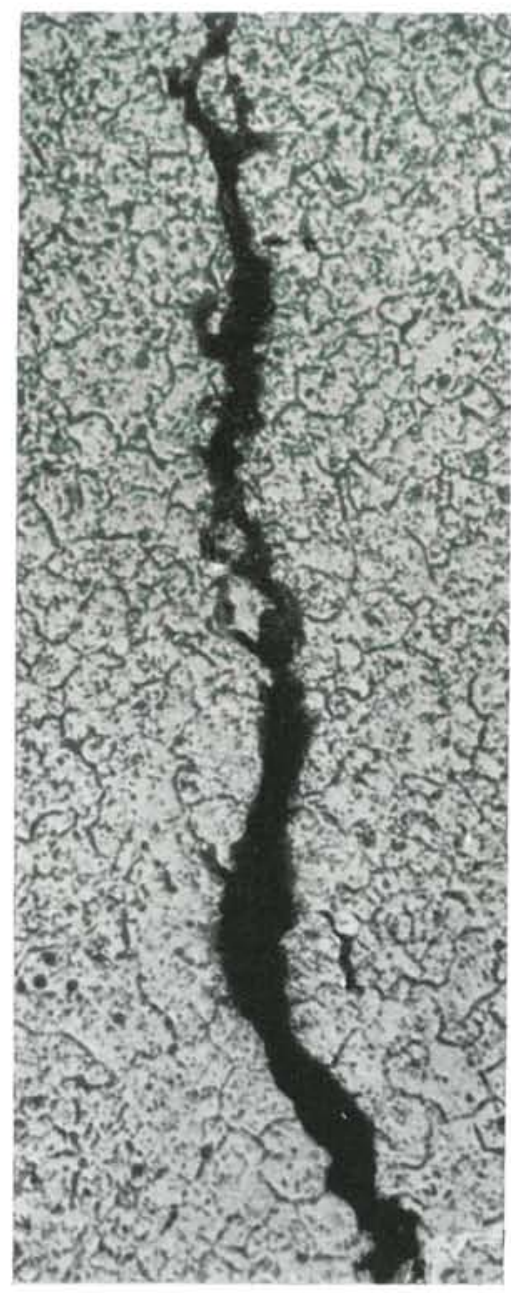

Fig. 10. Grieta producida por corrosión bajo tensión, debida al ataque de nitratos, en un acero templado $\mathrm{y}$ revenido. Aumentos $\times 300$.

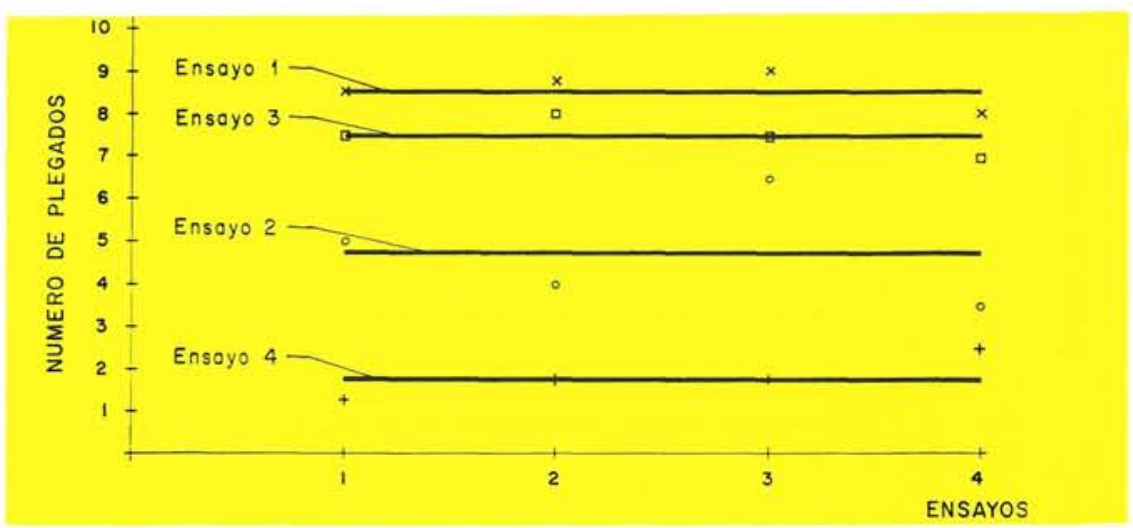

Fig. 11. Efecto de los nitratos, de la tensión y de la temperatura en la ductilidad. (Las características de los ensayos 1, 2, 3 y 4 se describen en la tabla 1 .)

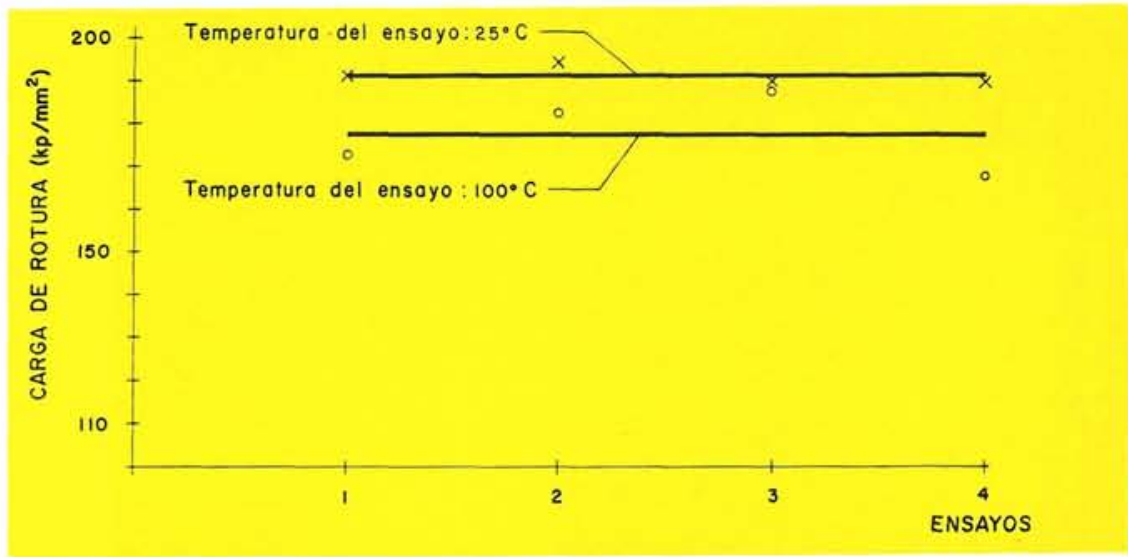

Fig. 12. Efecto de los nitratos y de la temperatura en la carga de rotura.-Tipo de cable: patentado $y$ trefilado. Tensión del alambre: $170 \mathrm{kp} / \mathrm{mm}^{2}$. Estos ensayos no han mostrado sintomas de corrosión bajo tensión. La ligera disminución de la $\sigma_{R}$ es debida a la mayor intensidad del ataque químico debido a la temperatura.

\section{4 \\ Eomellusiomes}

Los ensayos, iniciados en el Laboratorio Central, confirman el criterio de que los aceros patentados y trefilados reúnen excelentes condiciones para su uso en pretensado y que el fenómeno de corrosión bajo tensión debe ser poco frecuente en la práctica, si la obra está bien realizada.

Los agentes más agresivos son los que provocan fragilización por hidrógeno. El más frecuente es el $\mathrm{SH}_{2}$. Suele estar presente en atmósferas polucionadas por zonas industriales y puede formarse, también, a partir de sulfuros existentes en los materiales de construcción (cementos con alto contenido en alúmina y sulfuros). Menos frecuentes son los casos debidos a la formación de pares electroquímicos entre las cuñas de anclaje (o los encofrados de aluminio) y las armaduras, o los debidos a una protección catódica.

Los nitratos, citados frecuentemente como agentes típicos de corrosión bajo tensión, son menos peligrosos que el $\mathrm{SH}_{2}$. En aceros templados y revenidos (bonificados) se producen al poco tiempo roturas por corrosión bajo tensión debido a una solución caliente de nitratos, pero en los aceros patentados y trefilados no se ha observado ningún caso de rotura. No obstante, estos aceros pueden hacerse susceptibles mediante un tratamiento térmico que sea capaz de cambiar su estructura. Por ejemplo: Si se les calienta a $800^{\circ} \mathrm{C}$ y se templan a $300^{\circ} \mathrm{C}$ se forma una estructura bainítica muy sensible al agrietamiento por corrosión bajo tensión, no sólo de los nitratos, sino también a soluciones de cloruros alcalinos, soluciones de sulfatos o agua caliente [J. D. Gilchrist (1965)]. 


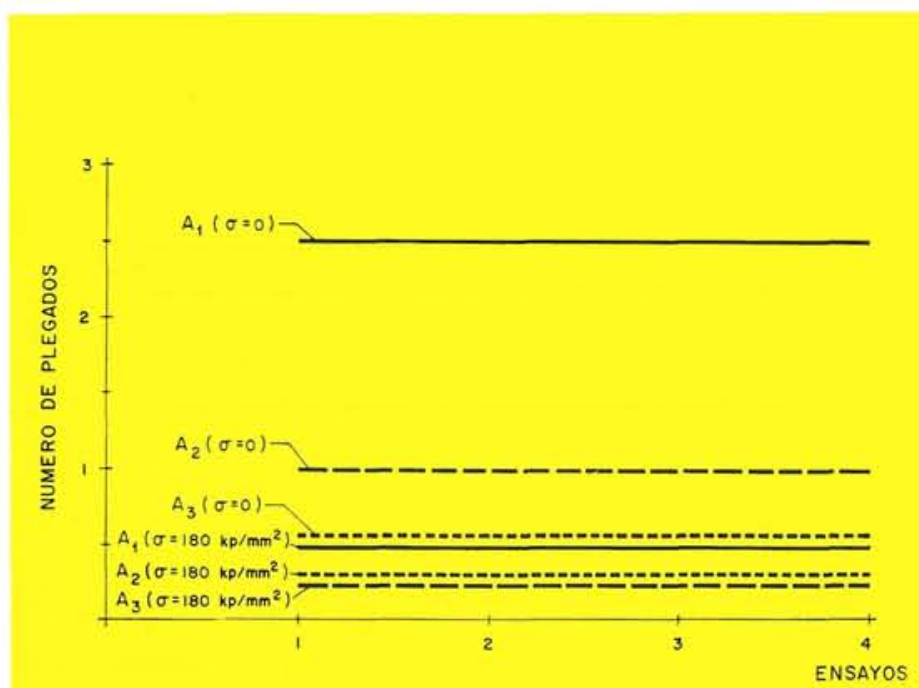

Fig. 13. Efecto del $\mathrm{SH}_{2} \mathrm{y}$ de la tensión en la ductilidad: La fragilización aumenta con la tensión del ensayo y con la falta de protección del acero ( $\mathbf{A}_{1}$ : Acero patentado $\mathbf{y}$ trefilado; $\mathbf{A}_{2}$ : Acero bonifi. cado; $\mathbf{A}_{3}:$ Acero decapado).

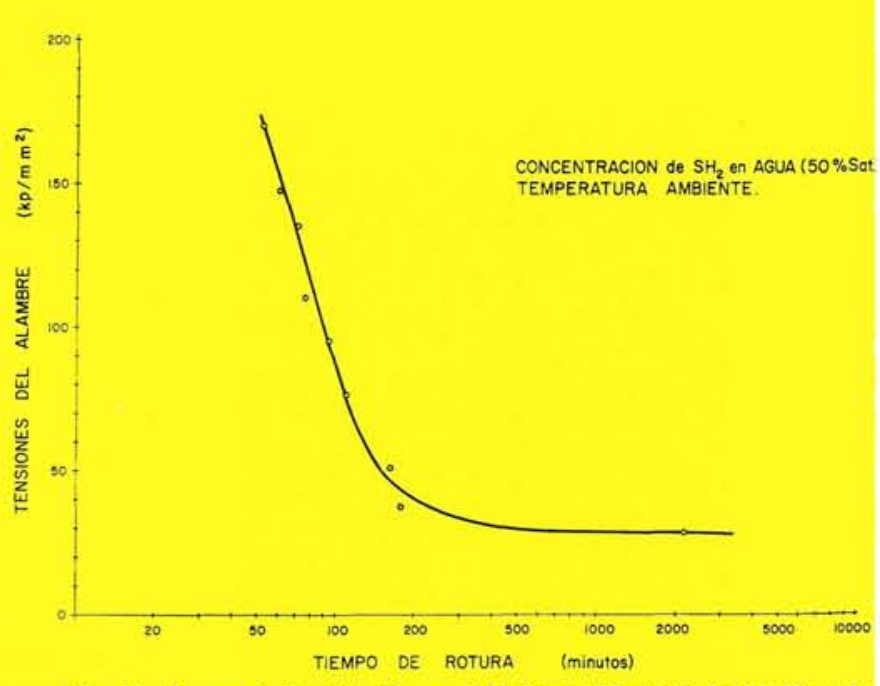

Fig. 14. Ensayo de fragilización por hidrógeno. Efecto de la tensión sobr el tiempo de rotura.
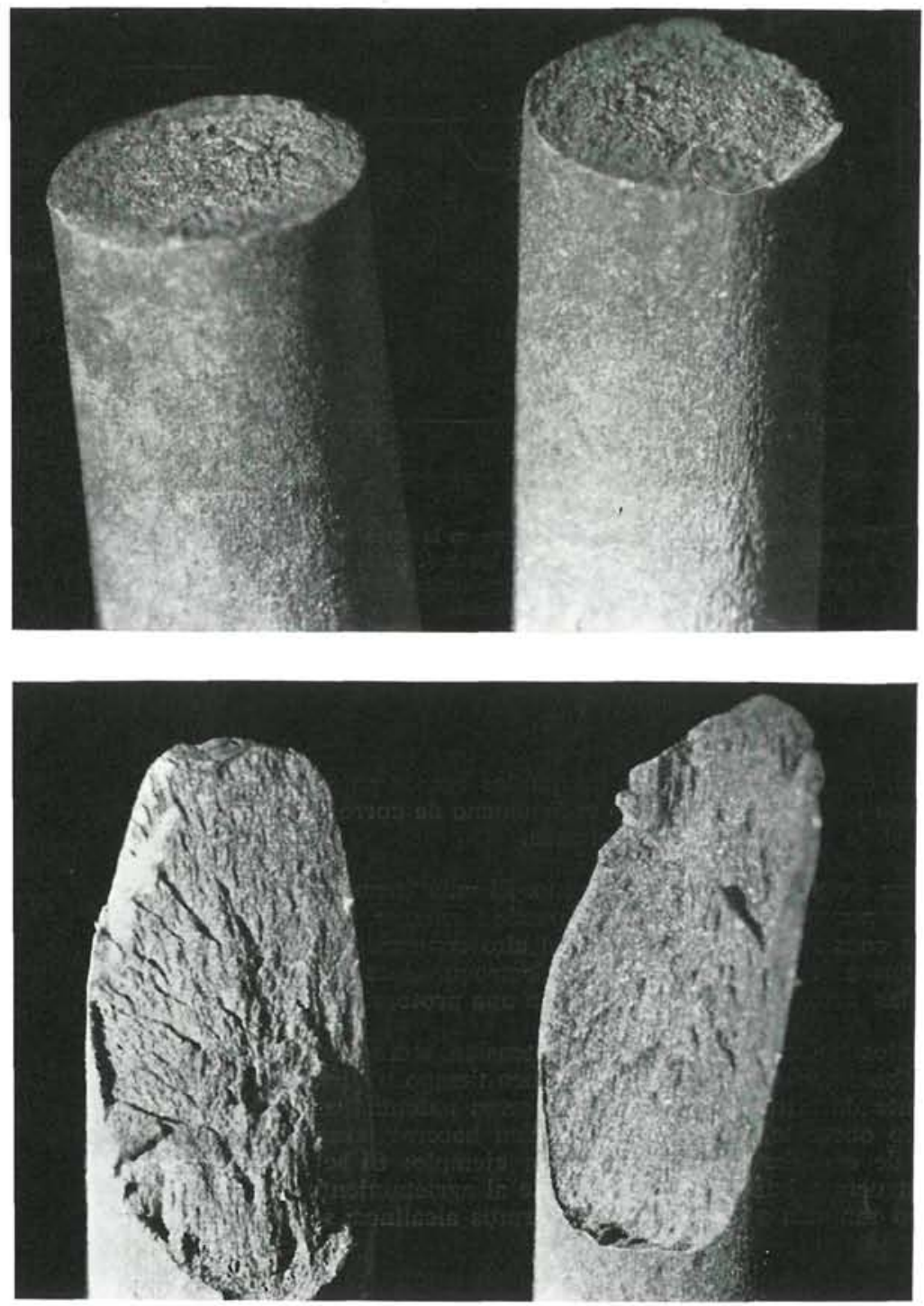

Los cloruros, menos peligrosos que los anteriores, son los causantes de un proceso de corrosión normal. Después de ensayos prolongados no se ha podido observar ninguna rotura por corrosión bajo tensión, a pesar de las drásticas alteraciones de la superficie (fig. 16). Solamente los alambres templados y revenidos han mostrado una notable fragilización. No debe olvidarse que los cloruros pueden producir picaduras profundas, cuyo efecto es más grave cuanto menor es el diámetro del cable.

Aunque en muchos artículos [M. Brachet, F. Duffaut y P. Lacombe (1967)] se citan casos de corrosión bajo tensión producidos por agentes aparentemente inertes (agua destilada o agua caliente), afortunadamente son casos raros que no se producen en los aceros patentados y trefilados cuando conservan todas sus propiedades y su superficie está en perfectas condiciones.

Con respecto al acero, parece que hay un acuerdo unánime en sancionar los aceros templados y revenidos, debido a su susceptibilidad a la corrosión, a pesar de que suelen tener buenas características mecánicas (la carga de rotura suele ser más alta que los patentados y trefilados). Ultimamente parece ser que se ha mejorado

Fig. 15. (Superior): Acero templado y revenido: Rotura por fragilización por hidrógeno. Aumentos $\times 12$. (Inferior): Acero patentado $\mathrm{y}$ trefilado: Rotura por fragilización por hidrógeno. Aumentos $\times 12$. 
la calidad de estos aceros mediante la orientación de los granos y una modificación de la composición química. Los ensayos realizados con este material indican un buen comportamiento frente a la corrosión [W. Jánicke, E. Stolte y H. Litzke (1965)].

Aunque los aceros patentados y trefilados son los de mayor garantía, se deben tener ciertas precauciones durante su almacenamiento, transporte y colocación. Durante el almacenamiento, no sólo debe evitarse el picado, sino la corrosión bajo tensión, producida por un ambiente agresivo y por tensiones de arrollamiento. No faltan ejemplos de roturas espontáneas durante el almacenamiento [J. J. Bouvy (1955) y R. Cigna (1965)].

Durante el transporte se debe evitar dañar la superficie de los alambres y, por último, durante su colocación deben extremarse las precauciones (fig. 17). El siguiente ejemplo es significativo: Durante la construcción de una tubería pretensada, la hilera por donde pasaba el cable estaba en malas condiciones, y provocó un recalentamiento del alambre.

El aumento de temperatura y el enfriamiento posterior, en el aire, provocaron un pequeño tratamiento térmico suficiente para susceptibilizarlo a la corrosión bajo tensión [R. E. Bald (1962)]. También puede ser perjudicial cuando está sin protección la excesiva permanencia en las vainas, tanto antes como después del tensado. Se ha sugerido que, además de las pruebas normales de obra, se hagan unos ensayos complementarios para determinar el comportamiento frente a la corrosión.

En el Laboratorio Central se han diseñado y se están poniendo a punto varias técnicas con objeto de lograr un ensayo sencillo y representativo de los fenómenos de corrosión bajo tensión y fragilización por hidró. geno que pueda completar los ensayos normales que se realizan para la caracterización de un acero.

Por último, se plantea la cuestión de la protección de las armaduras. Este problema tiene dos aspectos: el econó mico y el técnico [F. Dumas (1965)].

La protección de aceros mediante galvanizado es eficaz. Protege de los cloruros y la adherencia con el hormigón es buena. Sin embargo, entraña el peligro de fragilización por hidrógeno en los puntos donde se ha dañado el revestimiento de zinc (J. Voeltzel, G. Murry y A. Constant).

La utilización de resinas epoxi parece prometedora, ya que no sólo protege, sino que influye en la conservación del esfuerzo de pretensado [A. S. Hall (1962)]. Económicamente ambas soluciones son dudosas, ya que un hormigón bien ejecutado es una protección eficaz.

Se ha comprobado que un hormigón cuya composición granulométrica y ejecución aseguren un volumen mínimo de huecos, impide la corrosión de las armaduras [W. O. Everling (1954) y M. Duriez (1964)]. Actualmente no se corren riesgos usando aditivos de composición desconocida ni se superan las limitaciones en el uso de cloruros impuestas por las normas vigentes.

Fig. 16. Aspecto de la superficie de un alambre patentado y trefilado sometido a la acción de una solución de CINa saturada, durante 1 mes. Las fotografias se han obtenido con un microscopio electrónico de «SCANNING».

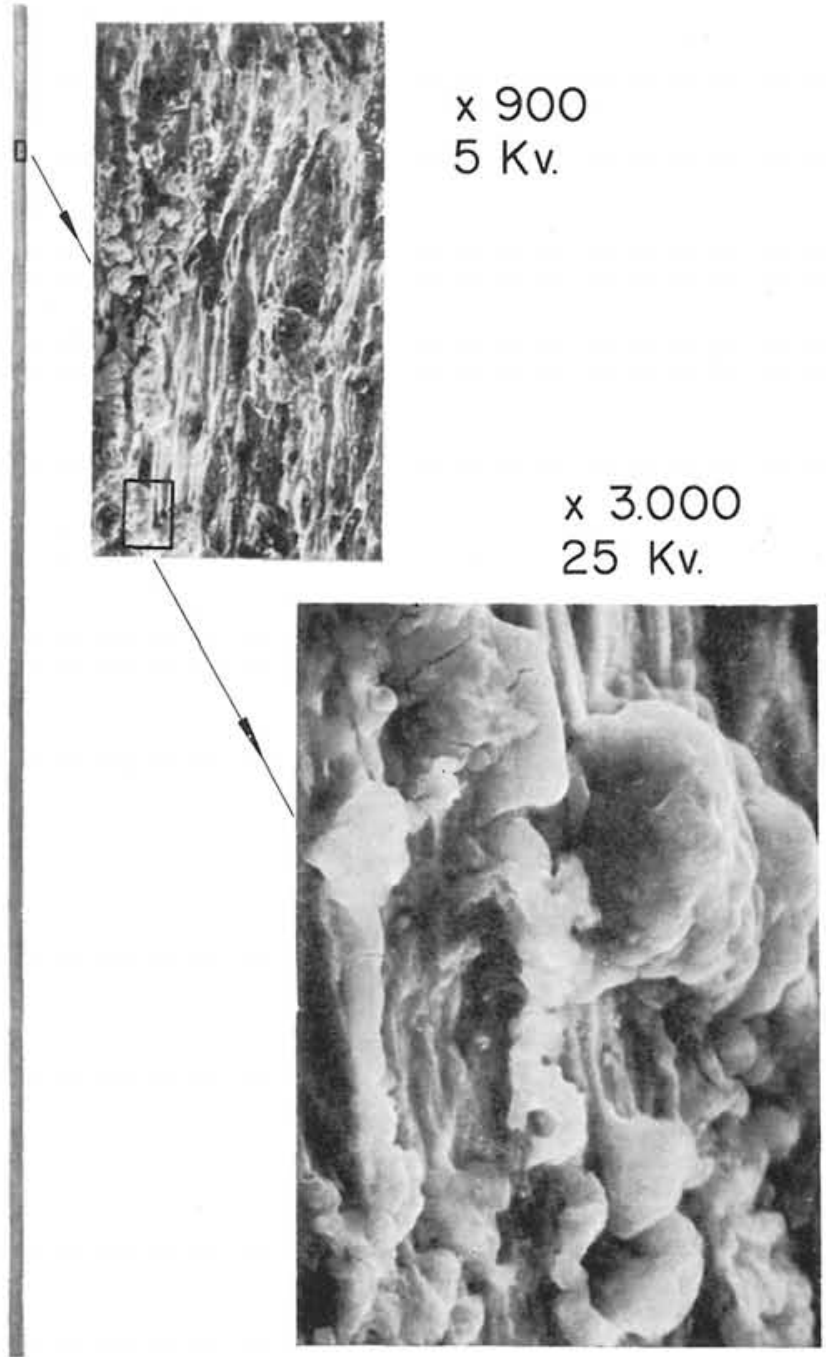


Fig. 17 a, b. Ejemplo de rotura por daños superficiales durante el almacenamiento, en un alambre para pretensado de $7 \mathrm{~mm}$ de diáme. tro: La entalladura que se produjo en la parte central causó la rotura frágil, en forma de "sombrero de Vikingon.
El espesor de los recubrimientos debe ser función del volumen de huecos del hormigón. Un revestimiento de $25 \mathrm{~mm}$ no es suficiente para un hormigón poroso y $20 \mathrm{~mm}$ pueden ser suficientes si el hormigón es muy compacto [Shütre y Kordina (1968)]. Sin embargo, el recubrimiento ha de ser lo suficientemente grande para asegurar que, durante el período de servicio de la estructura, la carbonatación no llegue a alcanzar el acero.

El proceso de carbonatación, producido al penetrar el $\mathrm{CO}_{2}$ a través del hormigón, es tanto mayor cuantas más fisuras existan. Por este motivo, las obras de hormigón pretensado están más expuestas a este proceso que debilita la protección del acero ofrecida por el $\mathrm{Ca}(\mathrm{OH})_{2}$. No existe un criterio que limite la anchura de las fisuras ni el tiempo de fisuración. Es un problema que está en estudio [S. Kajfasz (1968)].
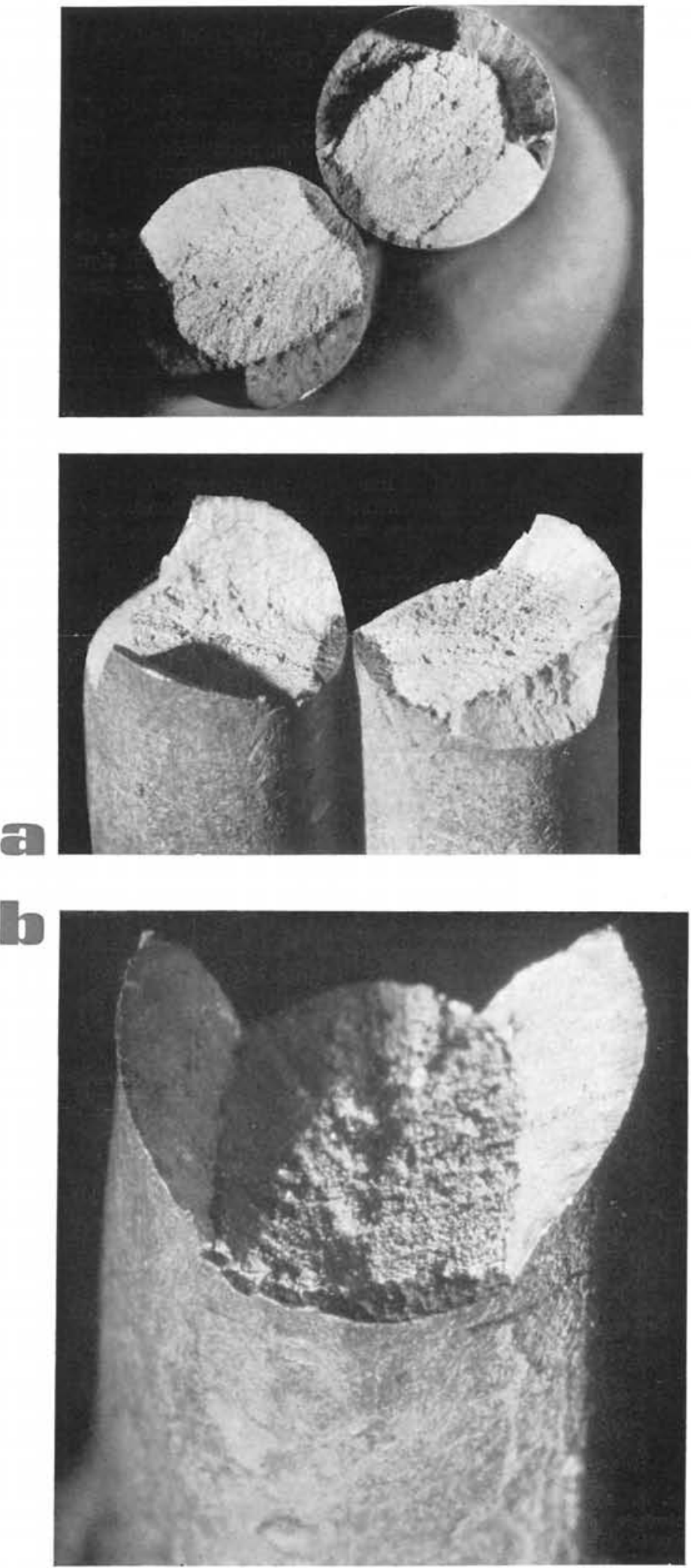


\section{Corrosion sous comtruinte des aciers pour précontrainte}

Florencio del Pozo, Dr. ingénieur des Ponts et Chausseés

Manuel Elices, Dr. ingénieur des Ponts et Chaussées

José Climent, licencié ès Sciences Physiques

Carlos Barba, Dr. ès Sciences Chimiques

Ce travail donne les premiers résultats d'un plan d'essais pour étudier le comportement face à la corrosion des aciers pour précontrainte, quí a été établi par le Laboratoire Central d'Essais des Matériaux de Construction du Centre d'Etudes et d'Expérimentation des Tra. vaux Publics. Les essais confirment le critère que les aciers brevetés et tréfilés remplissent de bonnes conditions pour leur emploi en précontrainte et
que le phénomène de corrosion sous contrainte doit être peu fréquent dans la pratique si l'ouvrage est bien réalisê.

Les agents les plus agressifs sont ceux qui provoquent la fragilisation par hydrogène, dont le plus fréquent est le $\mathrm{SH}_{\text {.. Les nitrates, cités }}$ très souvent comme étant des agents typiques de corrosion sous contrainte, sont moins dangereux que le $\mathrm{SH}_{2}$. Les aciers trempés et revenus sont plus sensibles à ces attaques que les aciers étirés à froid. Les chlorures ont résulté moins dangereux que les nitrates. Après des essais prolongés, il n'a pas été possible d'observer de ruptures par corrosion sous contrainte.

Le plan d'essais a pour but de parvenir à un essai simple et représentatif des phénomènes de corrosion sous contrainte et de fragilisation par hydrogène, capable de compléter les essais normalement exécutés pour la caractérisation d'un acier.

\section{Borrosion um dero stress im prestressimg streels}

Florencio del Pozo, Dr. civil engineer

Manuel Elices, Dr. civil engineer

José Climent, Physical Science graduate

Carlos Barba, Dr. in Chemistry. This paper summarises the first results of a testing program to investigate behaviour of prestressing steels in corrosion. This work
is being done at the Central Laboratory for Testing Construction Materials, of the Centre of Research and Experiments of Public
Works. Works.

The tests confirm the belief that patented drawn steels are very suitable for prestressing, and that the phenomenon of corrosion under stress must be infrequent in practice, if the work is well done.

The most aggressive agents are those which provoke fragilization through hydrogen, the most frequent being SH. Nitrates, which are tests no failure has been recorded due to corrosion under stress.

The purpose of this investigation is to develop a simple and representative test for the phenomenon of corrosion under stress and fragilization due to hydrogen, so that this test may complement the normal ones that are applied to classify steel.

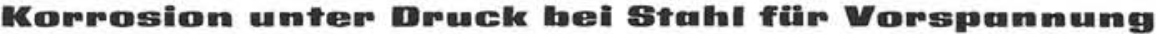

Florencio del Pozo, Dr. Tiefbauingenieur

Manuel Elices, Dr. Tiefbauingenieur

José Climent, Diplom.Physiker

Carlos Barba, Dr. in Chemie

In dieser Arbeit werden die ersten Ergebnisse eines Experimentierplans zusammengefasst zur Erforschung des Verhaltens der verschiedenen Stahlsorten für Vorspannung in Bezug auf Korrosion; diese Arbeit wird durchgeführt vom Laboratorio Central de Ensayos de Ma. (1) für öffentliche Bauarbeiten).

Die Versuche bestätigen, dass die patentierten und gezogenen Stahlsorten gute Bedingungen auf sich vereinigen für ihre Verwendung beim Vorspannen und dass das Phänomen der Korrosion unter Druck in der Praxis eigentlich nur selten auftreten dürfte, wenn die Bauarbeiten korrekt durchgeführt wurden.

Die agressivsten Wirkmittel sind diejenigen, die ein Brüchigwerden durch Wasserstoff hervorrunfen; das häufigste ist hier $\mathrm{SH}_{2}$. Die Ni. trate, die häufig als typisches Agens der Korrosion unter Druck zitiert werden, sind weniger gefährlich als $\mathrm{SH}_{2}$. Die gehärteten und verguteten Stahisorten sind hierfir anfaliger als kaltgezogener Stahi. Die Chiloride erwiesen sich als weniger gefährlich als die Nitrate. werden.

Ziel dieser Forschungsarbeiten ist es, einen einfachen und repräsentativen Versuch zu erstellen über die Phänomene der Korrosion unter Druck und des Brüchigwerdens durch Wasserstoff, in Ergänzung der normales Versuche, die durchgeführt werden zur Charakterisierung eines Stahls. 